\title{
Surface Bundles in Topology, Algebraic Geometry, and Group Theory
}
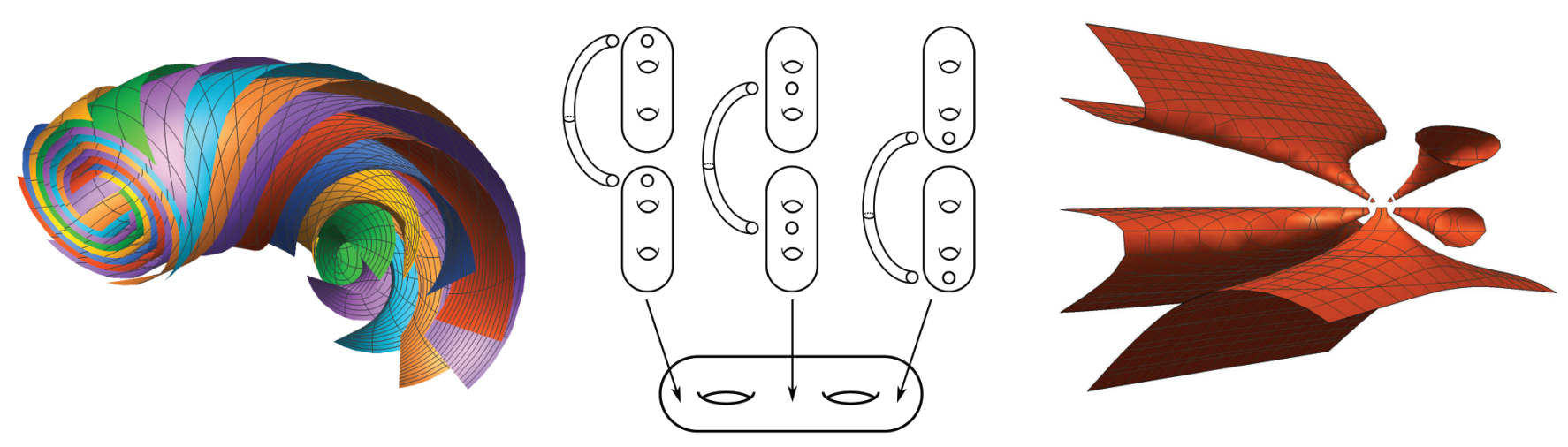

\section{Nick Salter and Bena Tshishiku}

\section{Surface Bundles}

A surface is one of the most basic objects in topology, but the mathematics of surfaces spills out far beyond its source, penetrating deeply into fields as diverse as algebraic geometry, complex analysis, dynamics, hyperbolic geometry, geometric group theory, etc. In this article we focus on the mathematics of families of surfaces: surface bundles. While the basics belong to the study of fiber bundles, we hope to illustrate how the theory of surface bundles comes into close contact with a broad range of mathematical ideas. We focus here on connections with three areas-algebraic topology, algebraic geometry, and geometric group theory-and see how the notion of a surface bundle provides a meeting ground for these fields to interact in beautiful and unexpected ways.

What is a surface bundle? A surface bundle is a fiber bundle $\pi: E \rightarrow B$ whose fiber is a 2-dimensional manifold

Nick Salter is a Ritt Assistant Professor at Columbia University. His email address is nks@math.columbia.edu.

Bena Tshishiku is an assistant professor at Brown University. His email address is bena_tshishiku@brown.edu.

Communicated by Notices Associate Editor Daniel Krashen.

For permission to reprint this article, please contact:

reprint-permission@ams.org.

DOI: https://doi.org/10.1090/noti2016
$S$ and whose structure group is the group $\operatorname{Diff}(S)$ of diffeomorphisms of $S$. In particular, $B$ is covered by open sets $\left\{U_{\alpha}\right\}$ on which the bundle is trivial $\pi^{-1}\left(U_{\alpha}\right) \cong U_{\alpha} \times S$, and local trivializations are glued by transition functions $U_{\alpha} \cap U_{\beta} \rightarrow \operatorname{Diff}(S)$.

Although the bundle is locally trivial, any nontrivial bundle is globally twisted, similar in spirit to the Möbius strip (Figure 1). This twisting is recorded in an invariant called the monodromy representation to be discussed in the section "Monodromy."

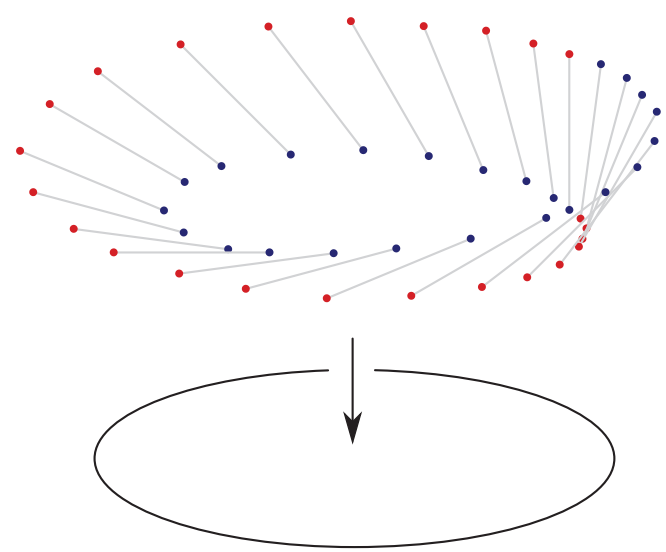

Figure 1. The Möbius strip is the total space of a bundle over $S^{1}$ whose fibers are diffeomorphic to $[0,1]$. 


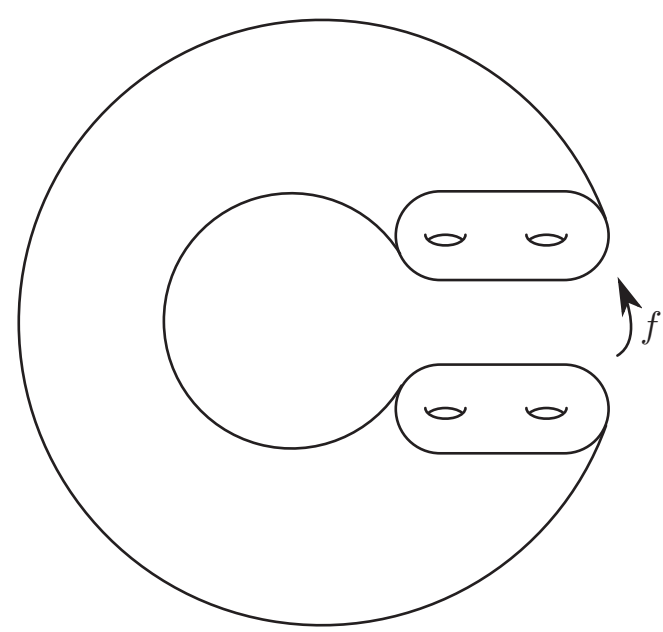

Figure 2. The mapping torus $E_{f}$ of a surface diffeomorphism $f: S \rightarrow S$. Note that the Möbius strip (Figure 1) can be constructed in a similar way.

A surface bundle $E \rightarrow B$ with fiber $S$ is also called an $S$-bundle over $B$, and $E$ is called the total space. Informally, one thinks of $E$ as a family of surfaces parameterized by $B$; i.e., for each $b \in B$, there is a surface $\pi^{-1}(b) \cong S$.

Surface bundles in nature. Surface bundles arise naturally across mathematics. The most basic source of $S$ bundles comes from the mapping torus construction. Given $f \in \operatorname{Diff}(S)$, define $E_{f}$ as the quotient of $[0,1] \times S$ by identifying $\{0\} \times S$ with $\{1\} \times S$ by $f$; then $E_{f}$ is the total space of an $S$-bundle over the circle $E_{f} \rightarrow S^{1}$. See Figure 2. Surprisingly, this simple-minded construction is ubiquitous in the classification of 3-manifolds and in particular hyperbolic 3-manifolds. Thurston proved that if $f$ is sufficiently complicated (pseudo-Anosov; cf. Theorem 2), then $E_{f}$ admits a hyperbolic structure, i.e., a Riemannian metric with sectional curvature $K \equiv-1$. Furthermore, by work of Agol, Wise, and Kahn-Markovic, every closed hyperbolic 3-manifold $M$ has a finite cover of the form $E_{f} \rightarrow M$ for some $f: S \rightarrow S$ [Ago13].

Surface bundles also figure prominently in 4-manifold theory. Donaldson [Don98] proved that every symplectic 4-manifold $M$ admits a Lefschetz fibration $M \rightarrow \mathbb{C} P^{1}$, which can be viewed as a surface bundle where finitely many fibers are allowed to acquire singularities of a simple form (so-called nodes).

Surface bundles appear in algebraic geometry, where they are more commonly known as families of curves. ${ }^{1}$ Special examples can be obtained by simply writing down families of equations. For instance, let $B$ be the space of tuples $b=\left(b_{1}, \ldots, b_{n}\right)$ of distinct points in $\mathbb{C}$, fix $d \geq 2$, and for $b \in B$, consider the surface

$$
S(b)=\left\{(x, y) \in \mathbb{C}^{2}: y^{d}=\left(x-b_{1}\right) \cdots\left(x-b_{n}\right)\right\} .
$$

\footnotetext{
${ }^{1}$ Since Riemann surfaces have complex dimension one, algebraic geometers refer to them as curves.
}

Then $E=\{(x, y, b) \mid(x, y) \in S(b)\}$ is the total space of an $S$-bundle over $B$ under the projection map $(x, y, b) \mapsto b$. Here $B$ is the configuration space of $n$ (ordered) points in $\mathbb{C}$. The study of this single $S$-bundle is already incredibly rich, with connections to representations of braid groups and geometric structures on moduli spaces of Riemann surfaces [McM13].

Vector bundles are also a source of surface bundles: given a rank-3 real vector bundle, the associated unitsphere bundle is an $S^{2}$-bundle. In fact, any $S^{2}$-bundle is obtained from this construction because, by a theorem of Smale, $\operatorname{Diff}\left(S^{2}\right)$ is homotopy equivalent to the orthogonal group $O(3)$ (this homotopy equivalence implies the bundle statement by the theory of classifying spaces discussed in "The Classification Problem"). On the other hand, if $S_{g}$ is a closed oriented surface of genus $g \geq 1$, then $\operatorname{Diff}\left(S_{g}\right)$ is not homotopy equivalent to a compact Lie group. As such, the study of $S_{g}$-bundles for $g \geq 1$ is the first instance of a nonlinear bundle theory. There are many analogies between the theory of vector bundles and surface bundles, but there are also many new phenomena, connections, and open questions.

Conventions. For the remainder of this article we assume, for simplicity, that $S=S_{\mathrm{g}}$ is a closed, oriented surface of genus $g \geq 1$ (and at times $g \geq 2$ ). Working with oriented surfaces, we consider only orientation-preserving diffeomorphisms; for brevity, we suppress this from the notation and will not mention it further.

The mapping class group. Given the wealth of examples of surface bundles described above, we need a good way to tell different surface bundles apart. We'll discuss two approaches to this-classifying spaces and monodromyin "The Classification Problem" and "Monodromy." Monodromy is a special feature for $S_{\mathrm{g}}$-bundles compared to other bundle theories, and it is where the mapping class group plays a prominent role.

To explain this, consider the mapping torus construction discussed above (Figure 2). If $f$ is isotopic to the identity (i.e., there is a path from $f$ to id in $\operatorname{Diff}\left(S_{g}\right)$ ), then $E_{f}$ is just the product bundle $S^{1} \times S_{\mathrm{g}}$. More generally, for any $f \in \operatorname{Diff}\left(S_{g}\right)$, the bundle $E_{f}$ is unchanged if $f$ is changed by an isotopy. Therefore, if we want to understand the different bundles obtained as mapping tori, we should start by considering the quotient $\operatorname{Mod}\left(S_{g}\right):=\operatorname{Diff}\left(S_{g}\right) / \operatorname{Diff}\left(S_{g}\right)$ by the (normal) subgroup of diffeomorphisms isotopic to the identity. The group $\operatorname{Mod}\left(S_{g}\right)$ is called the mapping class group. It is isomorphic to the group $\pi_{0} \operatorname{Diff}\left(S_{g}\right)$ of path components of $\operatorname{Diff}\left(S_{g}\right)$.

For example, $\operatorname{Mod}\left(T^{2}\right) \cong \mathrm{SL}_{2}(\mathbb{Z})$. Any $A \in \mathrm{SL}_{2}(\mathbb{Z})$ acts linearly on $\mathbb{R}^{2}$ and descends to $T^{2}$, and, conversely, up to homotopy or isotopy, a diffeomorphism of $T^{2}$ is determined by its action on $\pi_{1}\left(T^{2}\right) \cong \mathbb{Z}^{2}$. For $g \geq 1, \operatorname{Mod}\left(S_{g}\right)$ 
is an infinite, finitely presented group. In "Monodromy" we explain how $\operatorname{Mod}\left(S_{g}\right)$ plays a central role, not only for $S_{\mathrm{g}}$-bundles over $S^{1}$, but for $S_{\mathrm{g}}$-bundles over any base.

\section{The Classification Problem}

In this section we describe the basic tools and framework from algebraic topology for studying $S$-bundles. As mentioned above, we focus on the case $S=S_{g}$.

Two bundles $E \rightarrow B$ and $E^{\prime} \rightarrow B$ are isomorphic if there is a diffeomorphism $E \rightarrow E^{\prime}$ that sends fibers to fibers and covers the identity map on $B$.

Optimistically, one would like to solve the classification problem: for a given $B$, determine the set of isomorphism classes of $S_{\mathrm{g}}$-bundles $E \rightarrow B$. This problem can be translated to a homotopy-theoretic problem via classifying space theory.

Usually the classification problem is too difficult to solve completely. In practice one wants a rich collection of invariants that (i) measure topological properties of $S_{g}$ bundles and (ii) enable us to distinguish $S_{\mathrm{g}}$-bundles found in nature. In the study of vector bundles, a primary role is played by characteristic classes. Surface bundles also have a theory of characteristic classes, but as we explain, these are fairly coarse invariants.

Classifying space for surface bundles. For a CW-complex $B$, let $\operatorname{Bun}_{S_{g}}(B)$ be the set of isomorphism classes of $S_{g^{-}}$ bundles over $B$. For each $g \geq 0$, there is a space $\operatorname{BDiff}\left(S_{g}\right)$ and a bijection

$$
\operatorname{Bun}_{S_{g}}(B) \cong\left[B, \operatorname{BDiff}\left(S_{g}\right)\right]
$$

where the right-hand side is the set of homotopy classes of maps $B \rightarrow \operatorname{BDiff}\left(S_{g}\right)$. The space $\operatorname{BDiff}\left(S_{g}\right)$ is called the classifying space for $S_{g}$-bundles. In the language of homotopy theory, the functor $B \mapsto \operatorname{Bun}_{S_{g}}(B)$ is represented, and $\operatorname{BDiff}\left(S_{g}\right)$ is the universal element.

The space $\operatorname{BDiff}\left(S_{g}\right)$ is defined uniquely up to homotopy by the property that there is a principal $\operatorname{Diff}\left(S_{g}\right)$-bundle $P \rightarrow \operatorname{BDiff}\left(S_{g}\right)$ with $P$ contractible. In the bijection (2), given a map $B \rightarrow \operatorname{BDiff}\left(S_{g}\right)$, the corresponding $S_{g}$-bundle $E \rightarrow B$ is obtained by pullback:

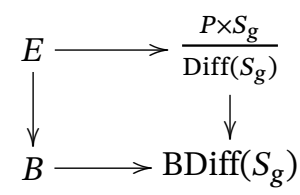

The bundle on the right is known as the universal $S_{\mathrm{g}}$-bundle. See [Mor01] for more details.

We want to understand the homotopy type of $\operatorname{BDiff}\left(S_{\mathrm{g}}\right)$. As mentioned above, there is a fibration $\operatorname{Diff}\left(S_{\mathrm{g}}\right) \rightarrow P \rightarrow$ $\operatorname{BDiff}\left(S_{g}\right)$ where $P$ is contractible. Hence the homotopy types of $\operatorname{Diff}\left(S_{g}\right)$ and $\operatorname{BDiff}\left(S_{g}\right)$ are closely related; indeed by the long exact sequence of homotopy groups,
$\pi_{i}\left(\operatorname{BDiff}\left(S_{g}\right)\right) \cong \pi_{i-1}\left(\operatorname{Diff}\left(S_{g}\right)\right)$. When $g \geq 2$, the homotopy type of $\operatorname{Diff}\left(S_{g}\right)$ is as simple as possible.

Theorem 1 (Earle-Eells). If $g \geq 2$, then the identity component $\operatorname{Diff}_{0}\left(S_{g}\right)<\operatorname{Diff}\left(S_{g}\right)$ is contractible. Consequently, the surjection $\operatorname{Diff}\left(S_{g}\right) \rightarrow \operatorname{Mod}\left(S_{g}\right)$ is a homotopy equivalence.

The homotopy type of $\operatorname{Diff}\left(S_{g}\right)$ for $g=0,1$ is also known: $\operatorname{Diff}\left(S^{2}\right)$ is homotopy equivalent to $O(3)$, and $\operatorname{Diff}\left(T^{2}\right)$ is homotopy equivalent to $T^{2} \rtimes \mathrm{SL}_{2}(\mathbb{Z})$; see e.g. [Mor01]. Theorem 1 was originally proved using complex analysis (Teichmüller theory) and PDE; a purely topological proof was given by Gramain (see [Hat]).

By Theorem 1, $\operatorname{BDiff}\left(S_{g}\right)$ is homotopy equivalent to $\operatorname{BMod}\left(S_{g}\right)$ for $g \geq 2$. Since $\operatorname{Mod}\left(S_{g}\right)$ is a discrete group, its classifying space is an Eilenberg-Mac Lane space $\operatorname{BMod}\left(S_{g}\right) \cong K\left(\operatorname{Mod}\left(S_{g}\right), 1\right)$. Observe that a map $f$ : $B \rightarrow \operatorname{BDiff}\left(S_{g}\right) \simeq \operatorname{BMod}\left(S_{g}\right)$ induces a homomorphism $\pi_{1}(B) \rightarrow \operatorname{Mod}\left(S_{g}\right)$. This is a fundamental invariant of the bundle associated to $f$, known as the monodromy; we discuss it further in "Monodromy."

In practice, it can be useful to have a concrete model for $\operatorname{BDiff}\left(S_{\mathrm{g}}\right)$. From the point of view of homotopy theory (as in [MW05, Hat]), the most useful model is the "Grassmannian" of surfaces embedded in $\mathbb{R}^{\infty}$. Unfortunately it would be too much of a detour to dwell on this further; see [Hat].

A second model for $\operatorname{BDiff}\left(S_{g}\right)$ is known as moduli space $\mathcal{M}_{g}$. Using Theorem 1 it suffices to give a model for $\operatorname{BMod}\left(S_{g}\right)$. For this we need a contractible space with a free, properly discontinuous action of $\operatorname{Mod}\left(S_{g}\right)$. To this end, consider the space $\mathcal{H}$ of hyperbolic metrics on $S_{g}$. The group $\operatorname{Diff}\left(S_{g}\right)$ acts by pullback of metrics, and $\operatorname{Diff}_{0}\left(S_{g}\right)$ acts freely. Miraculously, the Teichmüller space $\mathcal{T}:=\mathcal{H} / \operatorname{Diff}_{0}\left(S_{g}\right)$ is finite-dimensional and contractible: $\mathcal{T} \cong \mathbb{R}^{6 g-6}$. There is a natural action of $\operatorname{Mod}\left(S_{\mathrm{g}}\right)$ on $\mathcal{T}$, and the quotient $\mathcal{M}_{g}:=\mathcal{T} / \operatorname{Mod}\left(S_{g}\right)$ is the moduli space of hyperbolic metrics on $S_{g}$.

We would like to say that $\mathcal{M}_{g}$ is a model for $\operatorname{BMod}\left(S_{g}\right)$, but this is not true because $\operatorname{Mod}\left(S_{g}\right)$ does not act freely on $\mathcal{T}$. Indeed, the stabilizer of $[\mu] \in \mathcal{T}$ is the isometry group $\operatorname{Isom}\left(S_{g}, \mu\right)$, which is finite but not necessarily trivial. To circumvent this issue, we use the fact that $\operatorname{Mod}\left(S_{g}\right)$ contains many finite-index, torsion-free subgroups $\Gamma \leq \operatorname{Mod}\left(S_{g}\right)$. For such a group, $\mathcal{T} / \Gamma$ is a genuine $K(\Gamma, 1)$, and there is a finite covering $\mathcal{T} / \Gamma \rightarrow \mathcal{M}_{g}$ of orbifolds. For this reason, we call $\mathcal{M}_{\mathrm{g}}$ a virtual classifying space for $\operatorname{Mod}\left(S_{\mathrm{g}}\right)$. This is adequate for many purposes; e.g., there is an isomorphism

$$
H^{*}\left(\operatorname{BMod}\left(S_{g}\right) ; \mathbb{Q}\right) \cong H^{*}\left(\mathcal{M}_{g} ; \mathbb{Q}\right) .
$$

The moduli space $\mathcal{M}_{g}$ is many things at once. In addition to the set of hyperbolic metrics up to isometry, it is the set of algebraic curves up to isomorphism and the set of Riemann surfaces up to biholomorphism. This brings 
the study of $S_{\mathrm{g}}$-bundles into close contact with hyperbolic geometry, complex analysis, and algebraic geometry.

Characteristic classes. There are very few spaces $B$ for which $\operatorname{Bun}_{S_{g}}(B) \cong\left[B, \operatorname{BDiff}\left(S_{g}\right)\right]$ has been computed completely. Instead one can ask for invariants that distinguish different elements of $\left[B, \operatorname{BDiff}\left(S_{g}\right)\right]$.

A characteristic class for $S_{\mathrm{g}}$-bundles is a function $c$ that assigns to each $S_{\mathrm{g}}$-bundle $E \rightarrow B$ a cohomology class $c(E) \in H^{*}(B)$. In order to be useful, this function should be natural with respect to bundle pullbacks: given a pullback square

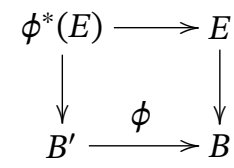

we require $c\left(\phi^{*}(E)\right)=\phi^{*}(c(E))$ in $H^{*}\left(B^{\prime}\right)$. Equivalently, a characteristic class is a natural transformation $c$ : $\operatorname{Bun}_{S}(\cdot) \rightarrow H^{*}(\cdot)$.

Since every $S_{\mathrm{g}}$-bundle $E \rightarrow B$ is obtained by pullback from the universal $S_{g}$-bundle over $\operatorname{BDiff}\left(S_{g}\right)$, any cohomology class $c \in H^{*}\left(\operatorname{BDiff}\left(S_{\mathrm{g}}\right)\right)$ defines a characteristic class; conversely, every characteristic class is of this form (evaluate on the universal bundle). In other words, $H^{*}\left(\operatorname{BDiff}\left(S_{g}\right)\right)$ is the set (or ring) of all characteristic classes of $S_{\mathrm{g}}$-bundles.

Computing $H^{*}\left(\operatorname{BDiff}\left(S_{g}\right)\right)$ is of fundamental importance for studying $S_{\mathrm{g}}$-bundles, but it is also of interest in other fields. By the preceding discussion,

$$
\begin{aligned}
H^{*}\left(\operatorname{BDiff}\left(S_{g}\right) ; \mathbb{Q}\right) & \cong H^{*}\left(\operatorname{BMod}\left(S_{g}\right) ; \mathbb{Q}\right) \\
& \cong H^{*}\left(\mathcal{M}_{g} ; \mathbb{Q}\right) .
\end{aligned}
$$

For our purpose, it is noteworthy that elements in the cohomology of $\operatorname{Mod}\left(S_{\mathrm{g}}\right)$ and $\mathcal{M}_{\mathrm{g}}$ give characteristic classes of $S_{\mathrm{g}}$-bundles.

Observe that the space $\operatorname{BDiff}\left(S_{g}\right)$, the group $\operatorname{Mod}\left(S_{g}\right)$, and the moduli space $\mathcal{M}_{g}$ are most naturally objects of algebraic topology, geometric group theory, and algebraic geometry, respectively. There has been a fertile exchange of ideas, tools, and techniques between these areas. To show this interaction, we briefly mention some of what is known about $H^{*}\left(\operatorname{BDiff}\left(S_{g}\right) ; \mathbb{Q}\right)$. Much of this is discussed in [Mor01] and references therein. The groups $H^{*}\left(\operatorname{BMod}\left(S_{g}\right)\right)$ satisfy homological stability, meaning that for each $i \geq 0, H^{i}\left(\operatorname{BMod}\left(S_{g}\right)\right)$ is independent of $g$ when $g \gg i$. This was proved by Harer in the early 1980s. Around the same time, Morita and Miller defined certain characteristic classes $e_{i} \in H^{2 i}\left(\operatorname{BDiff}\left(S_{\mathrm{g}}\right)\right)$, and Mumford defined analogous classes in the Chow ring of $\mathcal{M}_{\mathrm{g}}$. Collectively these are known as MMM classes or as $\kappa$ classes. Mumford conjectured that these classes generate the cohomology in degrees $i \ll g$, and this was proved in 2002 by Madsen-Weiss, who determined the homotopy type of $\operatorname{BDiff}\left(S_{g}\right)$ "in the limit" as $g \rightarrow \infty$ [MW05].
Despite all of this progress, $H^{*}\left(\operatorname{BDiff}\left(S_{g}\right) ; \mathbb{Q}\right)$ is still mostly unknown. By an Euler characteristic computation for $\mathcal{M}_{g}$ by Harer-Zagier, the MMM classes account for a small fraction of the total cohomology. We have only scratched the surface.

We conclude this section with a simple geometric argument that shows $H^{1}\left(\operatorname{BMod}\left(S_{g}\right) ; \mathbb{Z}\right)=0$ for $g \geq 3$. Recalling that $H_{1}(B G)$ is the abelianization $G^{\text {ab }}$, it suffices to show $\operatorname{Mod}\left(S_{g}\right)^{\mathrm{ab}}=0$. Dehn proved that $\operatorname{Mod}\left(S_{g}\right)$ is generated by mapping classes known as Dehn twists that are supported on an annulus in $S_{\mathrm{g}}$ whose complement is connected. Any two such Dehn twists are conjugate. ${ }^{2}$ Therefore, $\operatorname{Mod}\left(S_{\mathrm{g}}\right)^{\mathrm{ab}}$ is a quotient of $\mathbb{Z}$, generated by the image of any Dehn twist $A$. There is a relation $A B C=D E F G$ between seven Dehn twists known as the lantern relation (Figure 3 ). For $g \geq 3$ all seven annuli can be chosen to have connected complement, so that the image of this relation in $\operatorname{Mod}\left(S_{g}\right)^{\text {ab }}$ proves $3 A=4 A$ or $A=0$. This concludes the proof. See also [FM12, §5.1].

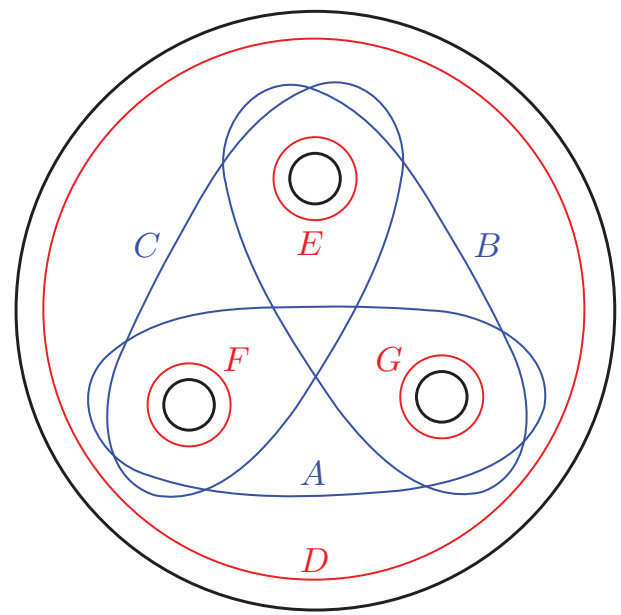

Figure 3. Dehn twists about these curves satisfy the lantern relation $A B C=D E F G$.

\section{Monodromy}

In "Surface Bundles" we saw that the mapping torus construction provides a rich supply of $S_{\mathrm{g}}$-bundles over $S^{1}$, but the argument of the preceding paragraph shows that none of these bundles are distinguished by characteristic classes! In this section we discuss the monodromy representation of an $S_{g}$-bundle. We will see that this is a complete invariant, so that in some sense we face the opposite problem: the challenge is to distill practical, computable information from the monodromy.

\footnotetext{
${ }^{2}$ To see this, cut $S_{g}$ along either annulus. By the classification of surfaces, the cut-open surfaces are seen to be homeomorphic. This homeomorphism can be extended via the identity across the annuli, yielding a map of $S_{g}$ taking one annulus to the other.
} 
Throughout this section we assume $g \geq 2$. By the bijection (2), associated to an $S_{g}$-bundle $E \rightarrow B$, there is a map $B \rightarrow \operatorname{BDiff}\left(S_{\mathrm{g}}\right)$, unique up to homotopy. The induced map on fundamental groups

$$
\pi_{1}(B) \rightarrow \pi_{1}\left(\operatorname{BDiff}\left(S_{g}\right)\right) \cong \pi_{0}\left(\operatorname{Diff}\left(S_{g}\right)\right) \equiv \operatorname{Mod}\left(S_{g}\right)
$$

is called the monodromy representation of $E \rightarrow B$.

The monodromy representation can be described concretely as follows: given $[\gamma] \in \pi_{1}(B)$ represented by $\gamma$ : $S^{1} \rightarrow B$, consider the pullback $\gamma^{*}(E) \rightarrow S^{1}$. Any bundle over the circle is obtained from the mapping torus construction (remove one fiber to get a bundle over the interval, which is trivial because any map $[0,1] \rightarrow \operatorname{BDiff}\left(S_{g}\right)$ is null-homotopic), so $\gamma^{*}(E) \cong E_{f_{\gamma}}$ for some $f_{\gamma} \in \operatorname{Diff}\left(S_{g}\right)$ whose isotopy class $\left[f_{\gamma}\right] \in \operatorname{Mod}\left(S_{g}\right)$ is independent of the choice of representative of $[\gamma]$. The monodromy representation is the map $[\gamma] \mapsto\left[f_{\gamma}\right]$. It measures how the "picture" of the fiber changes under the transition maps along the loop $\gamma$.

Monodromy as a complete invariant. By equation (2) and Theorem 1 for $g \geq 2$,

$$
\operatorname{Bun}_{S_{g}}(B) \cong\left[B, \operatorname{BDiff}\left(S_{g}\right)\right] \cong\left[B, \operatorname{BMod}\left(S_{g}\right)\right] .
$$

From $K(\pi, 1)$-theory, a map to $\operatorname{BMod}\left(S_{g}\right)$ is determined by the induced map on $\pi_{1}$, up to based homotopy. Hence $\left[B, \operatorname{BMod}\left(S_{g}\right)\right]$ is isomorphic to the quotient of $\operatorname{Hom}\left(\pi_{1}(B), \operatorname{Mod}\left(S_{g}\right)\right)$ by the action of $\operatorname{Mod}\left(S_{g}\right)$ by conjugation.

In summary, for $g \geq 2$ the isomorphism class of an $S_{g}$ bundle is determined uniquely by its monodromy representation. The monodromy is a complete invariant! Next we give examples of $B$ where this can be used to completely determine $\operatorname{Bun}_{S_{g}}(B)$.

As a trivial example, if $\pi_{1}(B)=0$, then the only $S_{g}$ bundle over $B$ is the trivial bundle $B \times S_{g}$. (Here it is important to remember that $g \geq 2$.) This illustrates a stark difference between $S_{g}$-bundles and vector bundles; for example, there are many nontrivial vector bundles over spheres $S^{k}$ with $k \geq 2$.

As a second example, for $B=S^{1}$, isomorphism classes of $S_{g}$-bundles over $S^{1}$ are in bijection with homomorphisms $\mathbb{Z} \rightarrow \operatorname{Mod}\left(S_{g}\right)$ up to conjugation, i.e., with conjugacy classes of elements of $\operatorname{Mod}\left(S_{g}\right)$. Here we clearly see why conjugation is relevant: to identify $E \rightarrow S^{1}$ with $E_{f}$, we must first choose a homeomorphism between the fiber over the basepoint and $S_{g}$. Different choices change $f$ by conjugation.

The surprising part of the statement "monodromy is a complete invariant" is that for any homomorphism $\rho$ : $\pi_{1}(B) \rightarrow \operatorname{Mod}\left(S_{g}\right)$, there is a bundle $E(\rho) \rightarrow B$ whose monodromy is $\rho$. It's not at all obvious how to explicitly construct $E(\rho)$ from $\rho$. This is the power of Theorem 1 . We note however that the monodromy is not a complete invariant of the total space up to homeomorphism, since a given 3-manifold may fiber as an $S_{\mathrm{g}}$-bundle in more than one way. See [Thu86].

The monodromy-topology dictionary. Let's think more about the bijection

$$
\operatorname{Bun}_{S_{g}}(B) \cong \operatorname{Hom}\left(\pi_{1}(B), \operatorname{Mod}\left(S_{g}\right)\right) / \text { conjugation. }
$$

In the previous section we gave examples where the lefthand side could be explicitly computed using the righthand side, but usually this is an unreasonable task. Even when $B=S_{h}$ is also a closed surface, there is no known classification of homomorphisms $\pi_{1}\left(S_{h}\right) \rightarrow \operatorname{Mod}\left(S_{g}\right)$.

We would like to emphasize a different perspective on (3) that leads to interesting problems. Observe that the left-hand side of (3) is topological, while the right-hand side is group-theoretic. Understanding how geometric or topological properties of $S_{\mathrm{g}}$-bundles translate to properties of the monodromy and vice versa leads to a dictionary. Below we mention a couple of entries of this dictionary.

Geometric classification of mapping tori. The precise conjugacy classification of elements of $\operatorname{Mod}\left(S_{g}\right)$ is well known. According to the Nielsen-Thurston classification, there are three types of conjugacy classes: periodic, reducible, and pseudo-Anosov. "Periodic" is synonymous with "finiteorder"; a reducible element preserves (setwise) some finite collection of curves up to isotopy. Thus a pseudo-Anosov element is simply any element with neither of these special properties. The miracle of the Nielsen-Thurston classification is that every pseudo-Anosov element nevertheless has a very tightly controlled form; see [FM12, \$13].

Thurston used this classification to describe the geometry of mapping tori.

Theorem 2 (Thurston). Fix $g \geq 2$ and fix $[f] \in \operatorname{Mod}\left(S_{g}\right)$. Then $[f]$ is

(a) periodic if and only if $E_{f}$ admits a Riemannian metric locally isometric to $\mathbb{U}^{2} \times \mathbb{R}$;

(b) reducible if and only if $E_{f}$ contains an incompressible torus;

(c) pseudo-Anosov if and only if $E_{f}$ admits a hyperbolic metric.

A geometric restriction on the bundle gives an algebraic restriction on the monodromy and vice versa. The most striking and difficult part of the theorem is: if $[f]$ is pseudoAnosov, then $E_{f}$ is hyperbolic. We remark that a mapping class can be both periodic and reducible, so (a) and (b) are not mutually exclusive.

Given Thurston's theorem, it is natural to ask for conditions on the monodromy of a bundle $E \rightarrow B$ with $\operatorname{dim} B \geq$ 2 that guarantee that $E$ has negative curvature. This seems to be a subtle question. It is not hard to see that it is necessary for every nontrivial element of the monodromy group 
to be pseudo-Anosov [FM02], but the converse is not generally known. It is a well-known open question whether or not there exists a homomorphism $\pi_{1}\left(S_{h}\right) \rightarrow \operatorname{Mod}\left(S_{g}\right)$ such that the image of every nontrivial element is pseudoAnosov.

Complex structures on $S_{g}$-bundles over surfaces. When $B=S_{h}$ is a closed surface, the total space $E$ of any $S_{g}$ bundle over $B$ is a compact 4-manifold and thus can potentially be diffeomorphic to a complex surface. Furthermore, it is possible for the bundle projection $E \rightarrow B$ to be holomorphic with respect to some complex structure on $B$. Since the monodromy $\rho$ of $E \rightarrow B$ determines the topology of $E$, this information is encoded inside $\rho$, albeit in a highly nontrivial way. In "Sections of $S_{\mathrm{g}}$-bundles," we will discuss the geometric Shafarevich problem, which shows that holomorphic families are exceedingly rare. Here, we mention some entries in the monodromy-topology dictionary concerned with the (non)existence of a complex structure on $E$.

Hodge theory provides one major source of obstructions. This is at its most powerful when the space under study is Kähler and not merely complex. It follows quickly from the Enriques-Kodaira classification that if $E$ is a compact complex surface that fibers over a surface, then $E$ is of general type and hence Kähler. Thus the basic "Kähler package" imposes nontrivial constraints on the cohomology algebra of $E$. By (3), the structure of $H^{*}(E ; \mathbb{Z})$ (as a ring) can be obtained from $\rho$. In fact, the cup product structure on an $S_{g}$-bundle is encoded as a certain family of characteristic classes with "twisted coefficients"; see [Sal18]. Another Hodge-theoretic obstruction is provided by Deligne's semisimplicity theorem, which places strong restrictions on how $\rho$ can act on the homology of the fiber [Del87].

To close this discussion we mention a theorem of Shiga [Shi97] providing another constraint on the monodromy of a holomorphic $S_{\mathrm{g}}$-bundle $E \rightarrow B$ over a compact Riemann surface $B$. Shiga's theorem asserts that in this setting, either all the fibers are biholomorphic or else the monodromy is geometrically irreducible, meaning that there is no simple closed curve globally fixed by the monodromy.

To further illuminate the themes under development (especially the monodromy-topology dictionary and interactions with algebraic geometry), in the final two sections we take a closer look at two topics: sections of $S_{g}$-bundles and $S_{g}$-bundles over surfaces.

\section{Sections of $S_{g}$-bundles}

A basic notion in any fiber bundle theory is that of a section: if $p: E \rightarrow B$ is a bundle map, then $s: B \rightarrow E$ is called a section if $p \circ s=\mathrm{id}$. In other words, a section is a continuously varying choice of distinguished point in each fiber. Given an $S_{\mathrm{g}}$-bundle $p: E \rightarrow B$ with corresponding monodromy representation $\rho: \pi_{1}(B) \rightarrow \operatorname{Mod}\left(S_{g}\right)$, there is a simple characterization of the homotopy classes of sections of $p$. Such sections are in correspondence with liftings $\tilde{\rho}$ of $\rho$ as encoded in the diagram below:

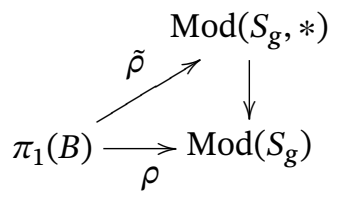

Here $\operatorname{Mod}\left(S_{g}, *\right)$ is the based mapping class group, defined as the group of diffeomorphisms fixing a distinguished point $* \in S_{g}$, modulo isotopies fixing $*$.

Sections of $S_{g}$-bundles in algebraic geometry and number theory. Before we discuss some of the tools used to construct and obstruct sections of $S_{\mathrm{g}}$-bundles, it is worthwhile to mention some applications. Sections of $S_{g}$ bundles are often of interest in problems of an algebrogeometric flavor. One notable instance of this concerns the geometric Mordell problem. Loosely speaking, this asks for an enumeration of holomorphic sections of $S_{\mathrm{g}}$-bundles over surfaces in the case where the total space has a complex structure. Arakelov and Parshin showed that the number of such sections is always finite. In fact, this is obtained from the geometric Shafarevich problem alluded to in "Monodromy." For simplicity we state the version obtained by Parshin; Arakelov treats the more general case when $B$ is a compact Riemann surface with finitely many points removed. See e.g. [McM00].

Theorem 3 (Geometric Shafarevich). Let B be a compact Riemann surface. For $g \geq 2$, there are only finitely many truly varying families $p: E \rightarrow B$ of Riemann surfaces of genus $g$.

A truly varying family $p: E \rightarrow B$ is an $S_{\mathrm{g}}$-bundle where $E$ has a complex structure, $p$ is holomorphic, and the fibers are not all biholomorphic. The geometric Mordell problem follows from geometric Shafarevich by way of the "Parshin trick." The idea is that each section $s: B \rightarrow E$ of a truly varying family can be used to construct a new truly varying family over $B$ by constructing a branched cover of $E$ branched along $s(B)$. This construction will be discussed further in "Bundles and Branched Covers" in the context of Atiyah-Kodaira bundles. Moreover, the genus of the fibers in the new family depends only on the genus of the original. Finiteness of families over $B$ (Shafarevich) then implies finiteness of sections (Mordell).

As explained by McMullen in [McM00], the geometric Mordell problem is actually the complex-geometric analogue of Faltings's theorem in number theory. Faltings's theorem concerns Diophantine equations $F(x, y, z)$ such as $x^{n}+y^{n}+z^{n}=0(n \geq 3)$ whose complex points determine a Riemann surface of genus at least 2; it asserts that such an equation has only finitely many rational solutions. Scheme-theoretically, one can view such a 
Diophantine equation as a "surface bundle" $\operatorname{over}^{3} \operatorname{Spec}(\mathbb{Z})$, where the "fibers" consist of the reductions of $F \bmod p$. From this point of view, a rational solution $(x, y, z)$ of $F$ determines a section of this bundle by assigning the distinguished point $(x, y, z)(\bmod p)$ to the fiber $F(\bmod p)$ over $p \in \operatorname{Spec}(\mathbb{Z})$. McMullen explains how Faltings's arguments have direct analogues in the setting of complex geometry, leading to the proof of the geometric Shafarevich problem given by Imayoshi-Shiga. In fact, the connections between $S_{g}$-bundles and number theory go beyond mere analogies. Recently, Lawrence-Venkatesh $[\mathrm{LV}]$ gave a new proof of Faltings's theorem that involves a topological analysis of the monodromy of certain $S_{\mathrm{g}}$-bundles over surfaces.

Sections of tautological bundles. Another application of the theory of sections of $S_{\mathrm{g}}$-bundles occurs in studying the existence and classification of sections of "naturally occurring" $S_{\mathrm{g}}$-bundles. The most "natural" of all such bundles is the universal curve $\mathcal{M}_{g, *} \rightarrow \mathcal{M}_{g}$ whose fiber over a point $x \in \mathcal{M}_{g}$ is the Riemann surface corresponding to $x$. The section question in this case simply asks if there is a way to continuously choose a distinguished point on all Riemann surfaces simultaneously. Unsurprisingly, $\mathcal{M}_{g, *} \rightarrow \mathcal{M}_{g}$ does not have a section for $g \geq 2$. However, it is possible to choose a continuously varying family of six everywhere-distinct points on the universal curve in genus 2, furnished by the so-called Weierstrass points (Figure 4). Thus a more sophisticated version of the section question
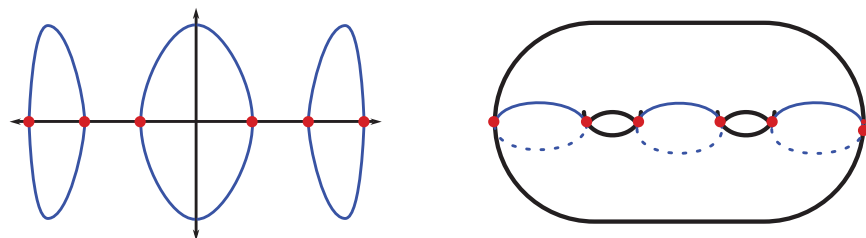

Figure 4. The blue set forms the real solutions of $y^{2}=-\left(x^{2}-1\right)\left(x^{2}-4\right)\left(x^{2}-9\right)$, plotted in $\mathbb{R}^{2}$ on the left. The red dots are the Weierstrass points. After projectivizing, the complex solutions are homeomorphic to a surface of genus 2 .

asks if it is possible to choose, for any $n \geq 1$, a "multisection" of $n$ everywhere-distinct points. If one restricts attention to holomorphic multisections, work of Hubbard [Hub76] shows that this is impossible, but this does not preclude the possibility that some merely continuous multisection could exist. For the universal curve $\mathcal{M}_{g, *}$, it was only recently shown that no continuous multisection exists for $g \geq 4$ by L. Chen and the first author [CS], building off ideas of Mess. The basic tool is the theory of canonical reduction systems, described below, which can be viewed as a version of the Jordan normal form for mapping classes.

Sections: Toolkit. The study of sections of $S_{g}$-bundles again incorporates themes and tools from a variety of mathematical disciplines. A first question is whether a given bundle admits any sections at all. Unlike in the theory of vector bundles, where the "zero-section" provides a quick affirmative answer to this question, an $S_{\mathrm{g}}$-bundle may or may not admit a section. This is similar to the situation one encounters when studying nowhere-vanishing sections of vector bundles. The standard machinery in the latter setting is obstruction theory, which manufactures cohomological invariants that obstruct the existence of sections. However, obstruction theory breaks down when the fibers are $K(\pi, 1)$ spaces with $\pi$ a group with trivial center, as is the case for $S_{g}$-bundles. Thus, by and large, the study of sections of $S_{g}$-bundles takes on a quintessentially geometric-group-theoretic flavor governed by the study of liftings $\tilde{\rho}$ as in (4).

Given $\rho: \pi_{1}(B) \rightarrow \operatorname{Mod}\left(S_{g}\right)$, how could one obstruct or classify the lifts $\tilde{\rho}: \pi_{1}(B) \rightarrow \operatorname{Mod}\left(S_{g}, *\right)$ ? The theory of canonical reduction systems provides one approach. Here we provide only a casual overview of how arguments using these ideas work; for a more precise discussion (including an actual definition of a canonical reduction system), see e.g. [FM12, \$13.2]. In keeping with the basic philosophy of geometric group theory, the method is to consider the action of $\pi_{1}(B)$ on the set of simple closed curves on $S_{g}$ afforded by the monodromy $\rho$. If one finds an ample supply of "simple" elements in the image of $\rho$ (e.g. elements with large centralizers in $\operatorname{Mod}\left(S_{g}\right)$ ), one can profitably understand the dynamics of this group action from the point of view of how $\pi_{1}(B)$ shuffles around simple closed curves on the surface. This information can be used to classify and obstruct sections: one asks where a distinguished point could be placed in relation to the simple closed curves under study, and in favorable circumstances one can see (e.g. by exploiting relations in $\pi_{1}(B)$ and/or $\operatorname{Mod}\left(S_{g}\right)$ ) that there is simply no place to put a distinguished point that is compatible with the known dynamics of the action.

One shortcoming of this approach is that current techniques apply only when $B$ has a fundamental group with certain properties. In many common situations (e.g. when $B=S_{h}$ is itself a surface), there are not enough commuting elements of $\pi_{1}(B)$ to be able to implement the above ideas. Our knowledge of sections of $S_{g}$-bundles over surfaces is extremely limited-in fact, the question of Mess [Kir78, Problem 2.17] from 1990 asking if every $S_{g}$-bundle over a surface admits a multisection is still open.

\section{Bundles and Branched Covers}

The main goal of this section is to describe a construction due to Atiyah and Kodaira of $S_{g}$-bundles over surfaces obtained by branched coverings. In contrast to the effortless way that $S_{g}$-bundles over $S^{1}$ are constructed (Figure 2), constructing interesting $S_{\mathrm{g}}$-bundles over surfaces takes work,

\footnotetext{
${ }^{3}$ For simplicity we are ignoring issues of good/bad reduction.
} 
and the branched covering constructions we discuss here have many interesting applications.

Before we begin, we mention that the bundle $E \rightarrow B$ over the configuration space from (1) in "Surface Bundles" is obtained via branched covers: the map $S(b) \ni(x, y) \mapsto$ $x \in \mathbb{C}$ is a $d$-fold cover branched over $b_{1}, \ldots, b_{n}$. Thus $B$ is parameterizing a family of branched covers of $\mathbb{C}$ with moving branched points. The Atiyah-Kodaira construction works similarly.

Atiyah-Kodaira bundles. We start with the basics of the construction, which we explain in one of the simplest cases. Consider the surface $S_{3}$, and let $\sigma: S_{3} \rightarrow S_{3}$ be a free involution (Figure 5). The product $S_{3} \times S_{3}$ contains a (disconnected) surface $\Sigma$, defined as the union of the graphs of the identity and $\sigma$. We would like to take a 2-fold cover $E \rightarrow S_{3} \times S_{3}$, branched over $\Sigma$.

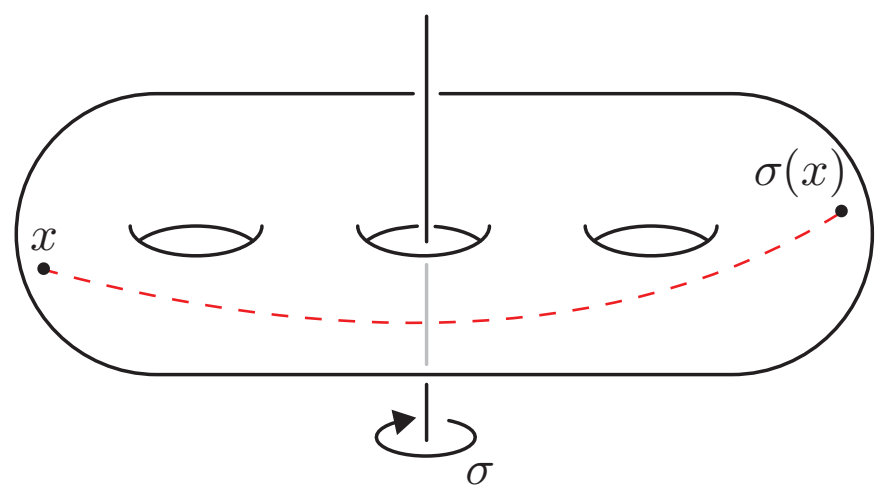

Figure 5. Free involution on surface of genus 3. Cut along the dotted line and double to obtain a 2-fold branched cover $S_{6} \rightarrow S_{3}$.

Before explaining more details, let's skip ahead to the output: the construction produces an $S_{6}$-bundle $E \rightarrow S_{129}$.

Where do these numbers come from? For the fiber $S_{6}$, first observe that $\Sigma \subset S_{3} \times S_{3}$ meets $\{x\} \times S_{3}$ in two points $(x, x)$ and $(x, \sigma(x))$, so under a double cover $E \rightarrow S_{3} \times S_{3}$ branched over $\Sigma$, the preimage of $\{x\} \times S_{3}$ is a 2-fold cover $S_{6} \rightarrow S_{3}$ branched over two points.

Now we explain the base $S_{129}$. The issue is that the branched cover $E \rightarrow S_{3} \times S_{3}$ is not guaranteed to exist. A sufficient condition for the existence is that the homology class $[\Sigma] \in \mathrm{H}_{2}\left(S_{3} \times S_{3}\right)$ is even. ${ }^{4}$ Unfortunately, $[\Sigma]$ is not even. To fix this, we first pass to the $2^{6}$-sheeted cover $S_{129} \rightarrow S_{3}$ with deck group $H_{1}\left(S_{3} ; \mathbb{Z} / 2 \mathbb{Z}\right)$. The preimage of $\Sigma$ under $S_{129} \times S_{3} \rightarrow S_{3} \times S_{3}$ determines an even homology class, and $E$ is defined as a branched cover of $S_{129} \times S_{3}$.

This construction can be done very generally: given a surface bundle $E \rightarrow B$ over a manifold and a multisection-viewed as a codimension-2 submanifold $\Sigma \subset E$ that projects to $B$ as a covering space-after

${ }^{4}$ A class $x$ is even if $x=2 y$ for some other class $y$. replacing $B$ with a finite cover, there is a cover $E^{\prime} \rightarrow E$ branched along $\Sigma$. If $E \rightarrow B$ and $\Sigma \subset E$ are both holomorphic, then the resulting bundle is also holomorphic. This is the essence of the Parshin trick discussed in "Sections of $S_{\mathrm{g}}$-bundles."

The Atiyah-Kodaira examples exhibit many interesting phenomena, and they appear in surprisingly many situations. A variant of the Atiyah-Kodaira construction appears in the work of Lawrence-Venkatesh [LV] mentioned in "Sections of $S_{\mathrm{g}}$-bundles." We close by mentioning a sampling of other applications of the construction.

Signature. The total space $E$ of an Atiyah-Kodaira bundle is a closed, oriented 4-manifold and therefore has a signature $\operatorname{sig}(E)$, defined as the signature of the intersection form $H_{2}(E) \times H_{2}(E) \rightarrow \mathbb{Z}$. Under a branched cover, the signature is multiplied by the degree of the cover with a correction term that is proportional to the self-intersection number of the branching locus. Thus, although $\operatorname{sig}\left(S_{3} \times S_{129}\right)=0$, we have $\operatorname{sig}(E)=256$. These were the first examples constructed of $S_{\mathrm{g}}$-bundles over surfaces with nonzero signature.

Consequently, the MMM class $e_{1} \in H^{2}\left(\operatorname{BDiff}\left(S_{g}\right)\right)$ is nontrivial for $g=6$ (and hence for $g \geq 6$ by Harer stability). To see this, we remark that the function that assigns to an $S_{g}$-bundle $E \rightarrow S_{h}$ the value $\operatorname{sig}(E) \in \mathbb{Z}$ can be viewed as a characteristic class. Specifically, there is a homomorphism $H_{2}\left(\operatorname{BDiff}\left(S_{g}\right)\right) \rightarrow \mathbb{Q}$ that sends a cycle represented by $S_{h} \rightarrow \operatorname{BDiff}\left(S_{g}\right)$ to the signature of the associated bundle. This is well defined because signature is a cobordism invariant. From the Atiyah-Kodaira construction $[\mathrm{sig}] \neq 0$ in $H^{2}\left(\operatorname{BDiff}\left(S_{6}\right) ; \mathbb{Q}\right)$, and since $e_{1}=3 \cdot[\operatorname{sig}]$ (by Hirzebruch's signature theorem), we conclude $e_{1} \neq 0$. In fact, the class [sig] $\in H^{2}\left(\operatorname{BDiff}\left(S_{g}\right) ; \mathbb{Q}\right)$ is nontrivial and generates this group when $g \geq 3$.

Nontriviality of MMM classes. Morita generalized the preceding argument to prove that all the MMM classes are nontrivial. More precisely, for each fixed $i$, there is $g \gg i$ so that $e_{i} \neq 0 \in H^{2 i}\left(\operatorname{BDiff}\left(S_{\mathrm{g}}\right) ; \mathbb{Q}\right)$. He proved this by iterating the Atiyah-Kodaira construction: for example, given the Atiyah-Kodaira bundle $p: E \rightarrow S_{129}$, consider the pullback to an $S_{6}$-bundle $p^{*}(E) \rightarrow E$. This bundle has a tautological section over which one can branch; in this way Morita obtained a bundle over a finite cover of $E$ with $e_{2} \neq 0$. See [Mor01, \$4.4] for more details.

In other directions, the Atiyah-Kodaira construction has also been used to give examples of inequivalent symplectic structures on 4-manifolds [LeB00] and examples of CAT(0) metrics with no Riemannian smoothings [Sta15]. A variant of the construction has also been used to study the geography problem for symplectic 4-manifolds [BNOP19]. 
Conclusion. There are many ways to arrive at the theory of surface bundles: as a nonlinear bundle theory (algebraic topology), as a source for interesting 3- and 4dimensional manifolds (low-dimensional topology and geometric group theory), or as objects naturally arising from moduli of Riemann surfaces (algebraic geometry). Each area brings to surface-bundle theory its own collection of ideas and techniques. This leads to a rich interaction where questions in one area motivate results in another. The interactions that we have discussed above represent only a small fraction of what is known and what is left to be discovered.

ACKNOWLEDGMENTS. The contents of this article owe a deep intellectual debt to Benson Farb, who introduced both of us to the theory of surface bundles as graduate students and has continued to shape our perspective on the field. We would like to thank him for extensive comments on a preliminary draft; we are also grateful to Lei Chen, Dan Margalit, and Shigeyuki Morita for their input.

\section{References}

[Ago13] Agol I. The virtual Haken conjecture, Doc. Math. (18):1045-1087, 2013. With an appendix by Agol, Daniel Groves, and Jason Manning. MR3104553

[BNOP19] Beyaz A, Naylor P, Onaran S, Park D. From automorphisms of Riemann surfaces to smooth 4-manifolds, Math. Res. Lett., to appear, 2019.

[CS] Chen L, Salter N. The Birman exact sequence does not virtually split, arXiv preprint, https://arxiv.org/abs /1804.11235 2018.

[Del87] Deligne P. Un théorème de finitude pour la monodromie, Discrete Groups in Geometry and Analysis (New Haven, Conn., 1984), Birkhäuser Boston, 1987, 1-19, DOI 10.1007/978-1-4899-6664-3_1 MR900821

[Don98] Donaldson SK. Lefschetz fibrations in symplectic geometry, Doc. Math. (Extra Vol. II):309-314, 1998. MR1648081

[FM12] Farb B, Margalit D. A Primer on Mapping Class Groups, Princeton Mathematical Series, vol. 49, Princeton University Press, Princeton, NJ, 2012. MR2850125

[FM02] Farb B, Mosher L. Convex cocompact subgroups of mapping class groups, Geom. Topol. (6):91-152, 2002, DOI 10.2140/gt.2002.6.91. MR1914566

[Hat] Hatcher A. A short exposition of the Madsen-Weiss theorem, https://arxiv.org/abs/1103.5223 2011.

[Hub76] Hubbard JH. Sur les sections analytiques de la courbe universelle de Teichmüller, Mem. Amer. Math. Soc., no. 166 (4):ix+137, 1976, DOI 10.1090/memo/0166 MR0430321

[Kir78] Kirby R. Problems in low dimensional manifold theory, Algebraic and Geometric Topology (Proc. Sympos. Pure Math., Stanford Univ., Stanford, Calif., 1976), Amer. Math. Soc., 1978, 273-312 MR520548
[LV] Lawrence B, Venkatesh A. Diophantine problems and $p$ adic period mappings, https://arxiv.org/abs/1807 $.02721,2018$.

[LeB00] LeBrun C. Diffeomorphisms, symplectic forms and Kodaira fibrations, Geom. Topol. (4):451-456, 2000, DOI 10.2140/gt.2000.4.451 MR1796448

[MW05] Madsen 1, Weiss M. The Stable Mapping Class Group and Stable Homotopy Theory, European Congress of Mathematics, 2005, 283-307 MR2185751

[McM00] McMullen CT. From dynamics on surfaces to rational points on curves, Bull. Amer. Math. Soc. (N.S.), no. 2 (37):119-140, 2000, DOI 10.1090/S0273-0979-99-008563. MR1713286

[McM13] McMullen CT. Braid groups and Hodge theory, Math. Ann., no. 3 (355):893-946, 2013, DOI 10.1007/s00208-012-0804-2 MR3020148

[Mor01] Morita S. Geometry of Characteristic Classes, Translations of Mathematical Monographs, vol. 199, American Mathematical Society, Providence, RI, 2001. Translated from the 1999 Japanese original; Iwanami Series in Modern Mathematics. MR1826571

[Sal18] Salter N. Cup products in surface bundles, higher Johnson invariants, and MMM classes, Math. Z., no. 3-4 (288):1377-1394, 2018, DOI 10.1007/s00209-017-19384 MR3779001

[Shi97] Shiga H. On monodromies of holomorphic families of Riemann surfaces and modular transformations, Math. Proc. Cambridge Philos. Soc., no. 3 (122):541-549, 1997, DOI 10.1017/S0305004197001825 MR1466656

[Sta15] Stadler S. An obstruction to the smoothability of singular nonpositively curved metrics on 4-manifolds by patterns of incompressible tori, Geom. Funct. Anal., no. 5 (25):1575-1587, 2015, DOI 10.1007/s00039-015-0341-8 MR3426062

[Thu86] Thurston WP. A norm for the homology of 3manifolds, Mem. Amer. Math. Soc., no. 339 (59):i-vi and 99-130, 1986. MR823443

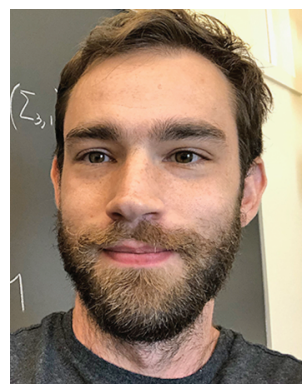

Nick Salter

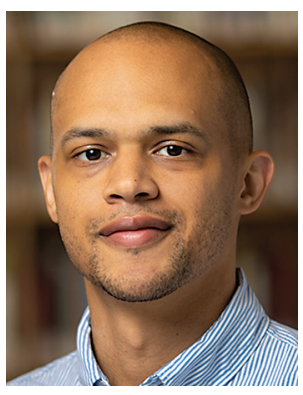

Bena Tshishiku

\section{Credits}

Figures 1-5 and opener image are courtesy of the authors.

Photo of Nick Salter is courtesy of Nick Salter.

Photo of Bena Tshishiku is courtesy of Nick Dentamaro/ Brown University. 